\title{
Publisher's Note: Glass-Glass Transitions by Means of an Acceptor-Donor Percolating Electric-Dipole Network [Phys. Rev. Applied 8, 054018 (2017)]
}

Le Zhang, Xiaojie Lou, Dong Wang, Yan Zhou, Yang Yang, Martin Kuball, Michael A. Carpenter, and Xiaobing Ren

(Q) (Received 31 January 2018; published 15 February 2018)

DOI: 10.1103/PhysRevApplied.9.029901

This paper was published online on 9 November 2017 with an error in the first affiliation. The first affiliation should read as "Multidisciplinary Materials Research Center and Center for Microstructure Science, Frontier Institute of Science and Technology and State Key Laboratory for Mechanical Behavior of Materials, Xi'an Jiaotong University, Xi'an, 710054, China." The affiliation has been corrected as of 1 February 2018. 